\title{
Damage detection in concrete precast slabs: a quick assessment through modal tests
}

\author{
Roberto Leal Pimentel ${ }^{1, a}$, Gabriel Soares Ferreira ${ }^{1}$, Márcio Santos Gonçalves ${ }^{1}$, Donald Steve Nyawako ${ }^{2}$, Paul Reynolds ${ }^{2}$ \\ ${ }^{1}$ Federal University of Paraíba, Department of Civil and Environmental Engineering, 58051-900 João Pessoa, Brazil. \\ ${ }^{2}$ University of Exeter, Vibration Engineering Section, College of Engineering, Mathematics and Physical Sciences, UK.
}

\begin{abstract}
The use of modal tests for detecting damage in reinforced concrete precast slabs is evaluated. A set of eight slabs were tested, each belonging to flats constructed for residential use. Two groups of slabs were identified and, in each group, both cracked and uncracked slabs were found. This made it possible to compare the responses of the slabs when subjected to modal tests. The tests were carried out employing an instrumented hammer and heel drops as excitation sources. Responses were measured using an accelerometer. The lowest natural frequencies of the slabs could be identified and after filtering the results, plots indicating the variation of the lowest natural frequency versus the number of cycles of free decay were obtained for each slab. Such a plot is of more general use than the value of the natural frequency by itself, as it does not depend on slab configuration. It was observed that the cracked slabs presented a similar pattern of variation of the natural frequencies throughout the decay, being distinctive from the pattern observed for their uncracked counterparts. This provided evidence that a quick assessment of the structural condition of such slabs through the use of the tests were feasible.
\end{abstract}

\section{Introduction}

Structural integrity assessment is a topic of major importance in civil structures. Some aspects that push structures to be evaluated are the possibility of overloading of structural members as well as potential design flaws.

Cracking is a condition that raises a flag regarding the integrity of a structural system, being a possible sign of reduction of structural strength. Visual inspection can be conducted to identify its presence. However, this is not efficient since one cannot properly assess the structural condition, and access to visual inspection may be impaired.

Use of modal testing can be considered a useful alternative to provide at least a qualitative assessment of the structural condition. In this context, a series of previous works related to this application can be found.

As an example, Pimentel et al. [1] investigated the structural behaviour of two slabs; one employing recycled aggregate concrete and another with conventional aggregate concrete, by subjecting both structures to progressive static loads (and thus generating cracking), applied in stages. A modal test was then carried out after each stage and dynamic properties (natural frequencies and damping ratios) were obtained and evaluated. In that work, results indicated that when cracked, both slabs presented similar reductions of stiffness in relation to the uncracked condition. Overall, it was concluded that modal testing was a useful complement of static testing, as a tool for comparing the features of different stages of cracking.

In the work of Russo [2], modal tests were performed in historic buildings for identification of their structural integrity. This author proposed a simplification of the testing procedure, employing excitations produced by ambient vibration and response measured by accelerometers. According to Russo [2], this proved to be an expeditious method for structural monitoring assessment.

Another related research comprises the evaluation of the extent of structural damage induced by natural phenomena through ambient vibration testing [3]. In that work, the changes in modal properties of a slab affected by a landslide were analysed. A comparison with the modal properties of a similar slab located in an upper floor within the same building was also carried out. Results indicated significant changes in natural frequencies and mode shapes. Irregularities in mode shape were correlated to the location of cracks in the affected structure.

Tan et al. [4] emphasized that reinforced concrete slabs are of particular interest because they are common structural elements of public structures (e.g. building floors, bridge decks, etc.). They performed vibration tests on reinforced concrete floor slabs in order to examine the use of strain gauges in the assessment of dynamic response during loading-induced damage of such

\footnotetext{
${ }^{\mathrm{a}}$ Corresponding author: r.pimentel@uol.com.br
} 
structures. The results in [4] showed that this testing approach worked for condition assessment of these structures.

Other research studies, such as the work of Júlio et al. [5] on the monitoring of the tower of the University of Coimbra, is also devoted to structural monitoring via modal identification.

It is worth noting that not only applications of modal tests for structural damage detection are being validated. The improvement of such a procedure has also been tackled by reducing errors inherent to the damage detection process [6].

Nowadays, precast slabs have a significant use in Brazil. Given the increasing demand of these structural elements, due to the faster building construction processes involved and, consequently, a great dynamism on the construction site [7], studies about the in-service structural behaviour of precast slabs are of interest.

In this work, the feasibility of modal testing as a damage detection method for precast slabs is experimentally investigated. In this sense, vibration tests were performed in eight concrete precast slabs intended for residential use. Modal properties were obtained.

Some of the slabs were cracked and others of the same group were uncracked. By comparing changes in natural frequency of similar cracked and uncracked slabs, the objective of this work is to explore the potential of modal testing for the identification of slabs with compromised structural integrity.

\section{Description of the case study}

Precast slabs of eight flats were investigated. The flats had similar architectural layout.

Each flat consists of a ground floor and a first floor with two rooms. The slab of one of these rooms was tested in each flat. Test equipment (computer, signal conditioners, power supplies, etc.) were installed in the other room.

In all flats, the slabs were constructed of reinforced concrete ribs and ceramic blocks, and a cast in place concrete topping.

In the absence of the design drawings, the initial stage of the investigation was to gather information about the geometry of the slabs, on site. Next, a visual inspection of the cracks was carried out, also locating them in the sketches being developed. Based on the geometric characteristics, slabs were divided into two groups, identified here as A and B. In both groups, cracked and uncracked slabs were found.

In sequence, test points were defined. It was done taking into consideration the geometry of the structure and the need to identify the first mode of vibration; it thus included the midpoint as well as points located at onethird of central and diagonal axes of the slab. Figures 1 to 6 contains the sketches, test points and illustrate the cracks observed in each slab. The points are identified as MID, LINE and SIDE, according to their position. In all cases, the cracks were seen underneath the slabs (bottom surface). The exception was Slab 1B, in which the crack was also seen on the top surface.

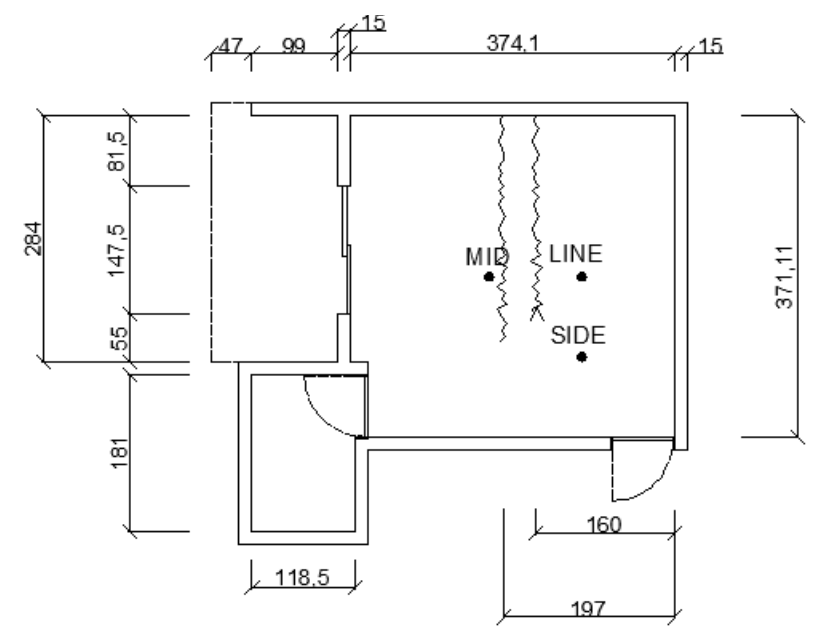

Figure 1. Sketch of the Slab 1A with representation of cracking and test points (dimensions in $\mathrm{cm}$ ).

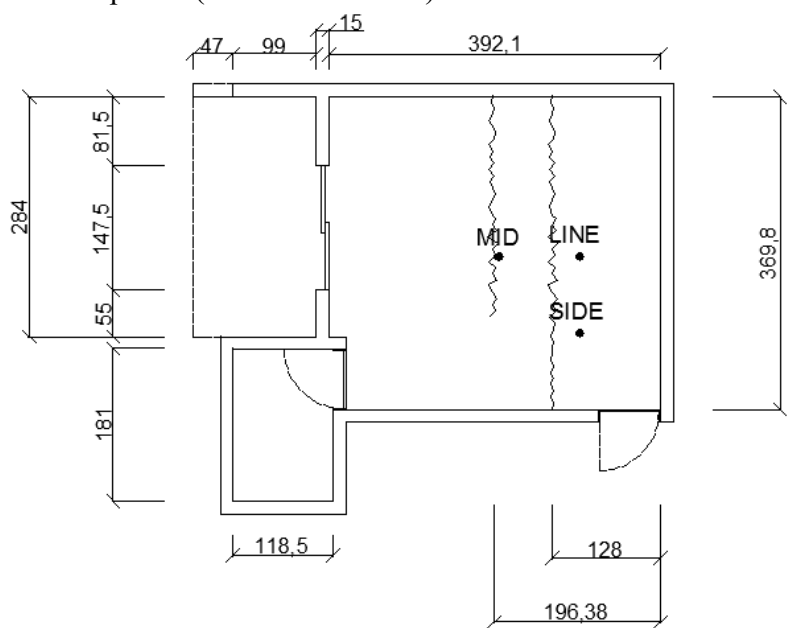

Figure 2. Sketch of the Slab 2A with representation of cracking and test points (dimensions in $\mathrm{cm}$ ).
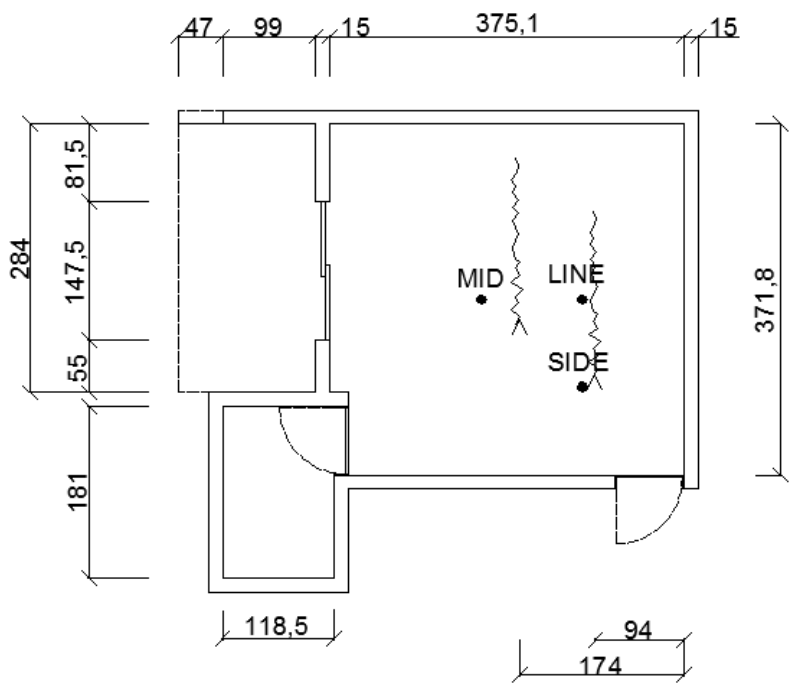

Figure 3. Sketch of the Slab 3A with representation of cracking and test points (dimensions in $\mathrm{cm}$ ). 


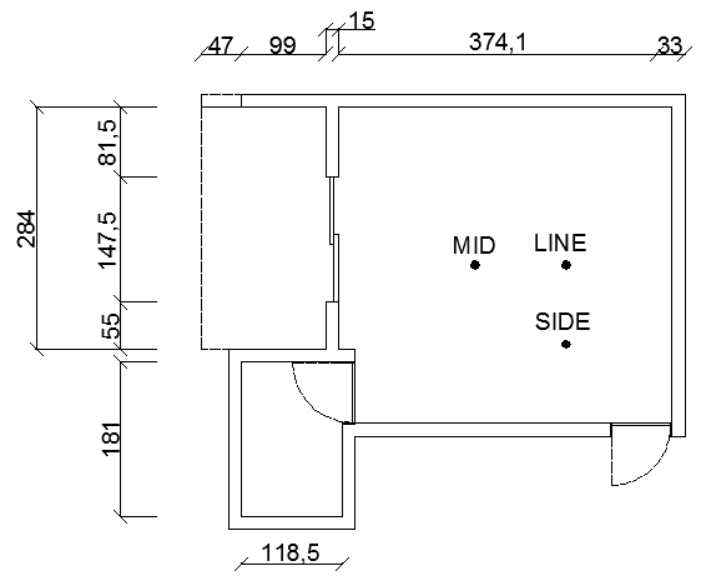

Figure 4. Sketch of the Slab 4A with representation of test points (dimensions in $\mathrm{cm}$ ). No cracks observed.

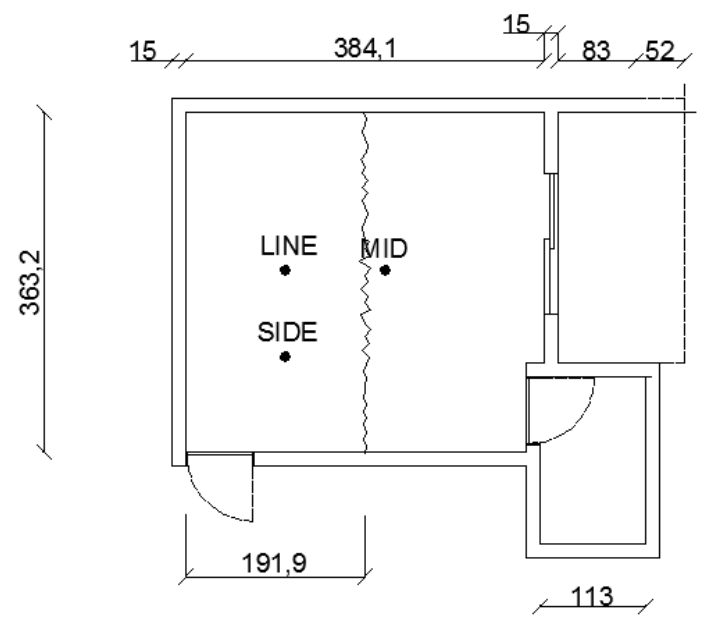

Figure 5. Sketch of the Slab 1B with representation of cracking and test points (dimensions in $\mathrm{cm}$ ).

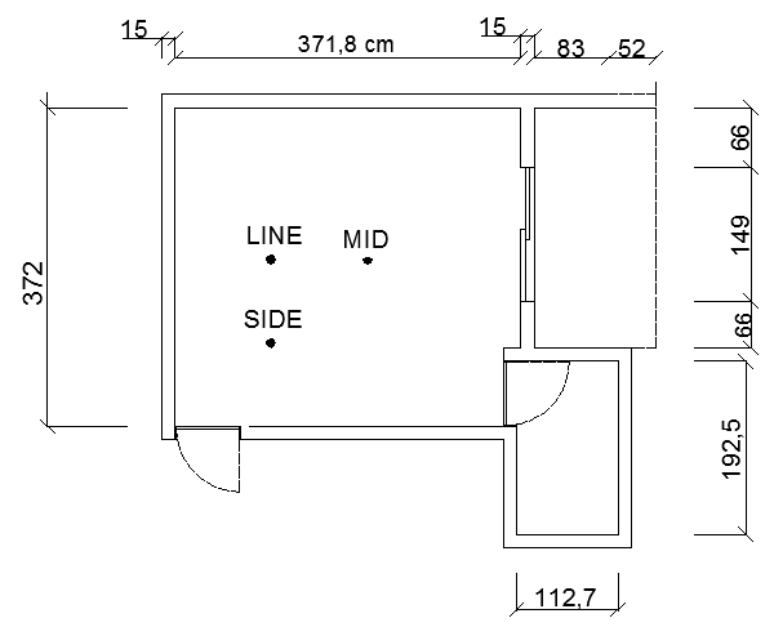

Figure 6. Sketch of the Slabs 2B to 4B with representation of test points (dimensions in $\mathrm{cm}$ ). No cracks observed.

Since cracking perpendicular to concrete ribs are more likely to be related to structural deterioration, the bottom cover of some slabs was removed to verify the position of the ribs with regard to the cracks. Since all the slabs had similar dimensions and all cracks were reasonably in the same position, the aforementioned procedure was done only in two slabs (1A and 1B). According to Figures 7 and 8, it was observed that cracks were developed at the interface between ceramic blocks and ribs, being thus parallel to the ribs.

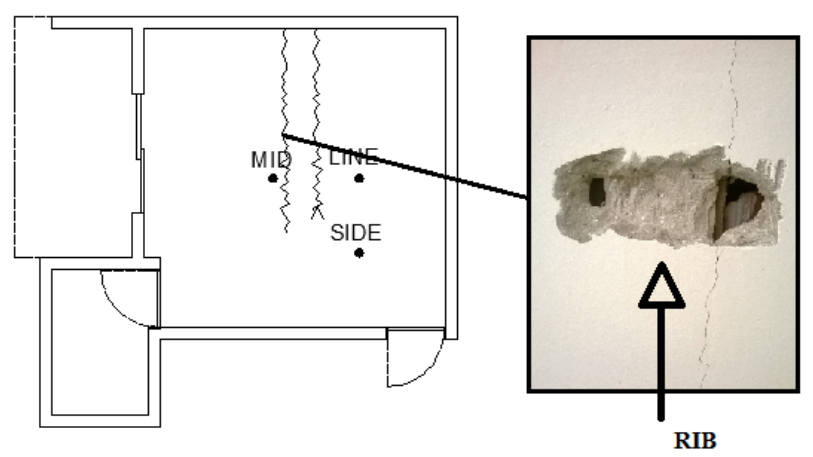

Figure 7. Detail of cracking, parallel to concrete ribs, on slab $1 \mathrm{~A}$.

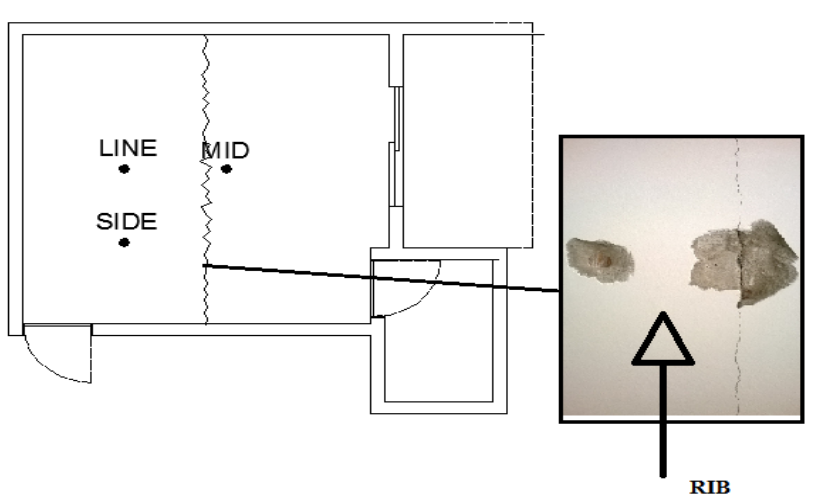

Figure 8. Detail of cracking, parallel to concrete ribs, on slab 1B.

\section{Methodology}

\subsection{Test equipment}

Excitations onto the slab were performed using an Instrumented B\&K Impact Hammer Model 8210, the sensitivity of which is $0.25 \mathrm{mV} / \mathrm{N}$. Additionally, heeldrop tests were carried out by three people.

An ENDEVCO Accelerometer Model 752A13, nominal sensitivity of $1 \mathrm{~V} / \mathrm{g}$, was used for capturing floor vibration response in different points of the slab. Both the accelerometer and the impact hammer were powered by ENDEVCO Signal Conditioners, Model 4416B.

Finally, the devices were connected to a Data Physics Spectrum Analyser Model Quattro that was in its turn connected to a personal computer. Frequency response functions, response spectrum and time domain signals were available for in situ analysis and also recorded for further processing.

\subsection{Testing procedure}

As mentioned previously, the initial stages were the visual inspection of cracks and preparation of sketches of the tested slabs.

The following stage was the execution of impact tests. During the tests with the instrumented hammer, one 
person operating the hammer remained seated on the tested floor and excited the structure, by hitting the hammer over a marked point. After each hit, the person remained still.

For the heel-drop tests, the same person that operated the hammer raised to their toes and dropped down to their heels to provide an impulsive excitation at a marked location on the floor.

An acquisition time of four seconds was adopted for both excitations. It corresponded to 4096 points of data capture, including 30 points before the beginning of the impacts, as pre-trigger points.

\subsection{Data processing}

Excitations on each marked point (either through impact hammer or heel-drop), in each tested slab, generated different data sets.

The acquired Frequency Response Function (FRF) data were processed to determine modes of vibration. Figure 9 presents a typical FRF, obtained from tests on Slab 1A, with its first vibration mode at $28 \mathrm{~Hz}$ clearly identified.

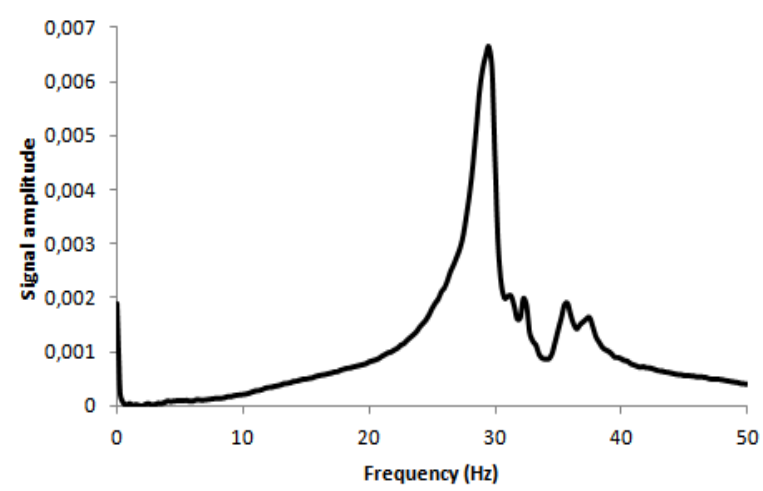

Figure 9. Example of the FRF obtained from Slab 1A.

The responses of the accelerometer in the time domain were selected for filtering. The filter was applied to obtain the response due to a single mode. In some response signals, this was not possible, due to closely spaced vibration modes with relevant participation in the response.

Figure 10 presents a typical filtered exponential decay, which was employed to obtain the natural frequency for successive cycles of vibration.

To get more accuracy in evaluating the natural frequencies from each vibration cycle, a linear interpolation was conducted among points of the time domain signal, to identify consecutive times corresponding to zeroes of the response. Through the difference between the time values obtained, the period of a given cycle was calculated and, from it, the frequency.

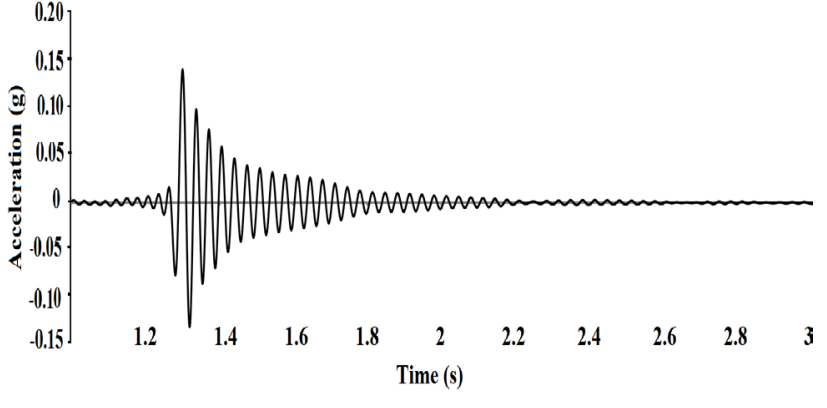

Figure 10. Exponential decay of the signal from a heel-drop test in Slab 1A. Both excitation and response were done at the middle of the slab.

To investigate the variation of the natural frequency along the filtered decay signal, a mean value of frequency throughout the signal is initially calculated. Then, the value obtained for the frequency in each cycle was divided by this respective mean frequency value. This also made it easy to compare the changes in frequencies for different slabs, cracked or uncracked.

Next, response spectra were plotted. A frequency range from 0 to $50 \mathrm{~Hz}$ was adopted, to cover the range of observed modes of the slabs. As the spectral peaks had different amplitude values among the test points, an average spectrum was obtained from the signals of the test points of each slab. In more details, the spectrum of each test point was first scaled, by dividing it by its respective maximum value of spectral peak. Then, an average spectrum of the spectra of all test points of a given slab was calculated. This procedure was repeated for all signals of each slab, making it easy to identify natural frequencies.

\section{Results and discussion}

The mean response spectra for each slab are shown in Figures 11 and 12, for each slab type. It was observed that slabs $1 \mathrm{~A}$ and $1 \mathrm{~B}$ had the lowest first natural frequencies ( both slabs were cracked). The slabs with no visible cracking possessed higher frequency values of the first mode.

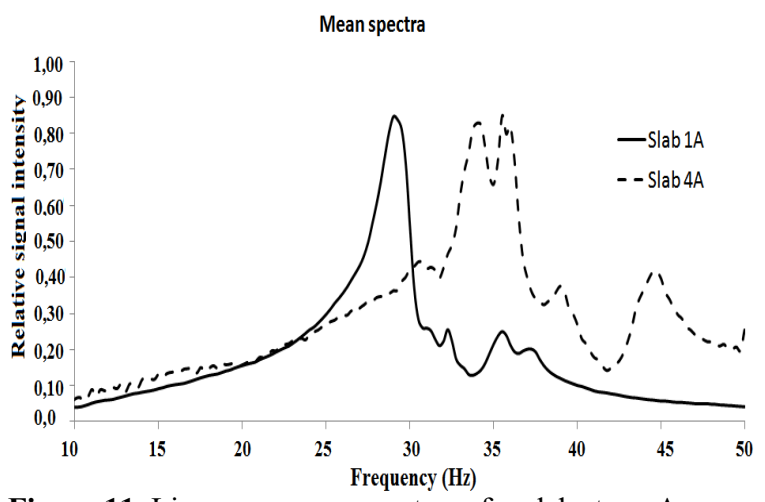

Figure 11. Linear response spectrum for slabs type A. 


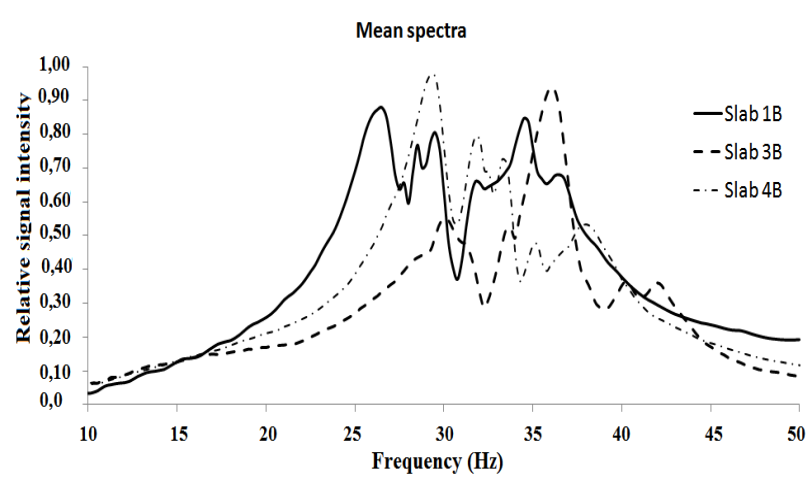

Figure 12. Linear response spectrum for slabs type B

For the analysis of variation of frequency along the decay plots, slabs of different groups (A and B) presented distinct frequencies, due to slightly different span dimensions.

For a comparison of results, all frequencies obtained from a given decay were divided by its respective maximum value, thus obtaining relative values. In sequence, a mean signal for the test points of each slab was obtained.

Plots are shown in Figures 13 and 14 for the observed changes in frequency along the signals. In Figure 13, it is worth noting that, for slab type $1 \mathrm{~A}$ (which is cracked), the frequency increases as number of cycles also does. For slab type 4A (uncracked), this behaviour is not observed.

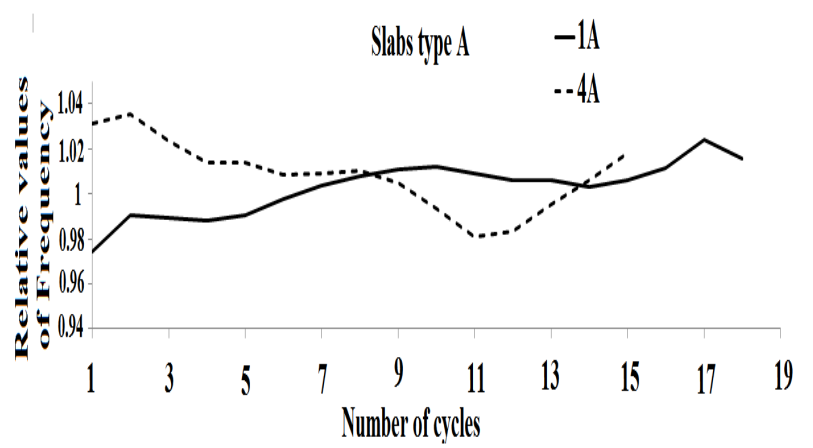

Figure 13. Changes of frequency along the decay for slabs type A.

For the slabs type B, a similar behaviour was observed. Slab 1B (cracked) reflected an increase in frequency along the decay, that is, with the number of cycles. Slab 4B (visually uncracked) presented a stable behaviour.

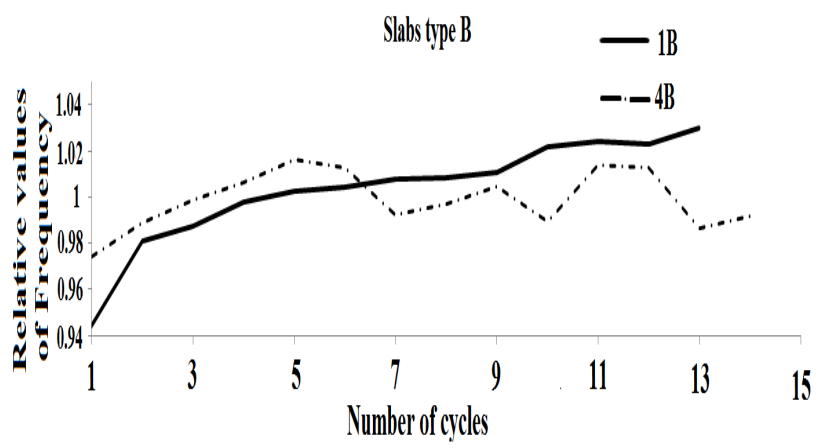

Figure 14. Changes of frequency along the decay for slabs type B.

\section{Concluding remarks}

In this work, two groups of four concrete precast slabs with (and without) cracks were investigated by means of modal tests, aiming to analyse the applicability of such tests in damage detection.

The procedures employed were based on changes on natural frequencies, considering that the occurrence of cracking would induce changes on the stiffness of the slabs.

The results showed that, for cracked slabs, apart from presenting low values of natural frequency than their uncracked counterparts, the changes on frequency along the decay signal showed a pattern not observed on the uncracked slabs. It was observed a systematic growth of the natural frequency along the decay signal of the cracked slab. Since the excitations were applied impacts, it can be concluded that the reduction of the level of induced displacements along the decay led to an increase in stiffness, possibly due to closing of cracks towards the end of the acquisition.

It is worth noting that the level of cracking presented by the slabs was relatively modest, found along a few contact lines between ribs and ceramic blocks. This may not characterize an induced structural damage, and might have arisen due to minor problems during the construction phase that do not seriously compromise the strength capacity of the slabs. In spite of this, the technique of observing the changes on natural frequency along the decay enabled the differences in pattern between cracked and uncracked slabs to be detected.

Since such behaviour along the decay does not depend on slab configuration, this constitutes a qualitative way of identifying cracked slabs, particularly in those cases in which visual inspection may be impaired.

\section{Acknowledgements}

The authors acknowledge both Brazilian institutions CNPq and CAPES, and the British Council (UK) through the Researcher Links programme, for the financial support for this research work.

\section{References}

1. R.L. Pimentel, S. M. Torres, E. T. Ferreira, A. B. de Melo. Structural Integrity Assessment of Precast Concrete Slabs Employing Conventional and Recycled Coarse Aggregates via Vibration Tests. Key Engineering Materials, 600, 504-513 (2014).

2. S. Russo. Using Experimental Dynamic Modal Analysis in Assessing Structural Integrity in Historic Buildings. The Open Construction and Building Technology Journal, 8, 357-368 (2014).

3. H. Hashim, Z. Ibrahim, H. A. Razak. Dynamic characteristics and model updating of damaged slab from ambient vibration measurements. Measurement, 46, 1371-1378 (2013).

4. H. C. Tan, O. O. R. Famiyesin, M. S. E. Imbabi. Dynamic deformation signatures in reinforced 
concrete slabs for condition monitoring. Computers and Structures, 79, $2413-2423$ (2001).

5. E. N. B. S. Júlio, C. A. da S. Rebelo, D. A. S. G. Dias-da-Costa. Structural assessment of the tower of the University of Coimbra by modal identification. Engineering Structures, 30, 3468-3477 (2008).

6. Q. Huang, P. Gardoni, S. Hurlebaus. A probabilistic damage detection approach using vibration-based nondestructive testing. Structural Safety, 38, 11-21 (2012).

7. M. N. Kataoka, M. A. Ferreira, A. L. H. C. El Debs, A study on the behavior of beam-colum connections in precast concrete structures: experimental analysis. Rev. IBRACON Estrut. Mater. [online], 5, 848-873 (2012). 